Para enlazar con este artículo / To link to this article:

http://dx.doi.org/10.6035/MonTI.2019.11.3

Para citar este artículo / To cite this article:

Cerezo Herrero, Enrique. (2019) "Sistematización de competencias en la enseñanza de lengua B para Traducción e Interpretación en el EEES a partir de un modelo de competencia traductora." In: Tolosa Igualada, Miguel \& Álvaro Echeverri (eds.) 2019. Porque algo tiene que cambiar. La formación de traductores e intérpretes: Presente \& futuro / Because something should change: Present $\&$ Future Training of Translators and Interpreters. MonTI 11, pp. 77-107.

\title{
SISTEMATIZACIÓN DE COMPETENCIAS EN LA ENSEÑANZA DE LA LENGUA B PARA TRADUCCIÓN E INTERPRETACIÓN EN EL EEES A PARTIR DE UN MODELO DE COMPETENCIA TRADUCTORA
}

\author{
Enrique Cerezo Herrero \\ enrique.cerezo@uchceu.es \\ Universidad CEU Cardenal Herrera
}

\section{Resumen}

El objetivo de este artículo es ofrecer un modelo de competencias para la enseñanza de la Lengua B en las titulaciones de Traducción e Interpretación dentro del Espacio Europeo de Educación Superior (EEES). Hasta la fecha, las asignaturas de lengua extranjera no han recibido la atención suficiente en el ámbito de la investigación y las directrices ofrecidas en documentos oficiales han sido muy vagas y generales y no han tenido en cuenta las necesidades formativas y profesionales de los discentes. Con el objeto de revertir esta situación resulta necesario establecer un modelo de competencias basado en la propia actividad traductora. Para ello, en este trabajo se tomará como referencia el modelo de competencia traductora del grupo PACTE. Se espera que la sistematización de competencias que aquí se propone sirva para arrojar un rayo de luz en una subdisciplina fundamental en la pedagogía de la traducción y proporcione a los docentes una base sólida sobre la naturaleza de las asignaturas de la Lengua B en las titulaciones de Traducción e Interpretación.

\begin{abstract}
"Systematization of competences in the teaching of Language B in Translation and Interpreting Studies within the EHEA taking as a basis a translation competence model".

The objective of this article is to offer a model of competences for the teaching of the Language B in Translation and Interpreting degrees within the European Higher Education Area (EHEA). To date, foreign language subjects have not received due attention in the field of research and the guidelines offered thus far in official documents have been very vague and general, not taking account of the training and professional
\end{abstract}


needs of the students. With the aim of reverting this situation, it is necessary to establish a competence-based model in accordance with translation practice. For this purpose, in this work we will take as a reference the Translation Competence Model proposed by PACTE group. The systematization of competences proposed in this paper is expected to shed light on a fundamental sub-discipline in translation pedagogy and provide lecturers with a sound foundation regarding the nature of Language B subjects in Translation and Interpreting degrees.

Palabras clave: traducción, lengua, competencia traductora, subcompetencias, formación

Keywords: translation, language, translation competence, subcompetences, training 


\section{Introducción}

Desde el año 2010, Europa se ha visto inmersa en un proceso de convergencia en materia educativa como consecuencia de la creación del Espacio Europeo de Educación Superior (EEES). Este proceso de cambio tiene como objetivo principal la armonización de la formación universitaria en toda la zona euro mediante la movilidad del estudiantado y profesorado. Para ello, ha sido necesario rediseñar y adecuar todas las titulaciones universitarias con el objeto de:

- Crear un sistema de titulaciones fácilmente comprensible y comparable que permita el reconocimiento de estudios entre distintas instituciones y países.

- Facilitar el acceso al mercado laboral entre países de la zona euro.

- Establecer un sistema universitario basado fundamentalmente en dos ciclos (Grado y Posgrado). ${ }^{1}$

- Establecer un sistema de transferencia de créditos ECTS (European Credit Transfer System) que favorezca la movilidad de docentes y discentes.

- Impulsar la cooperación educativa dentro del EEES y, de este modo, garantizar los estándares de calidad del mismo.

En lo que al plano metodológico se refiere, esta nueva concepción de la docencia universitaria ha supuesto (Cano 2008):

- Una profunda revisión de la función docente.

- Dejar atrás el papel del docente como transmisor de conocimiento para pasar a ser un facilitador de oportunidades de crecimiento.

- Integrar el qué se espera que los alumnos aprendan con el cómo y para qué.

- Reducir el número de clases magistrales donde predomina el conocimiento conceptual y fomentar un aprendizaje más participativo a partir del trabajo autónomo.

1. En este nivel se incluyen tanto los programas de Máster como de Doctorado. 
Dicho cambio supone una oportunidad institucional de renovación metodológica (De Miguel 2006; Bolívar 2008). Se trata de renovar, entre otros, los materiales docentes para que promuevan un aprendizaje efectivo y al amparo de las nuevas directrices europeas en materia educativa. Inicialmente, esta transformación se materializó a través de los libros blancos, los cuales ofrecían directrices concretas en cuanto a la implementación de los nuevos títulos universitarios. En el caso del Libro Blanco del título de Grado en Traducción e Interpretación, las competencias propuestas para las lenguas extranjeras son demasiado generales, siendo el profesorado el responsable de interpretarlas y aplicarlas a partir de su propio conocimiento y experiencia en actividades de traslación interlingüística. Sin embargo, en numerosas ocasiones, el profesorado que imparte lenguas proviene con frecuencia de estudios filológicos y no posee necesariamente conocimientos específicos sobre traducción. Es por ello que resulta necesario contar con un elenco detallado de competencias específicas que permita dar forma a su docencia según las necesidades lingüísticas y exigencias impuestas por la propia actividad traductora.

Pese a la creación del EEES y la necesidad de cambio metodológico de las titulaciones universitarias, puede afirmarse que los esfuerzos por desarrollar un modelo específico de competencias aplicable a la enseñanza de lenguas extranjeras dentro de las titulaciones de Traducción e Interpretación en España han sido escasos hasta le fecha. Esto se debe, entre otros aspectos, a la falta de investigación dentro de este campo, hecho que ya denunciaba Hurtado (1999a) a finales de la década de los años $90 \mathrm{y}$, por ende, a una falta de interés por parte de la comunidad científica hacia la formación lingüística del traductor o intérprete (Cerezo 2013). Sin embargo, las lenguas son el motor de toda actividad de base traductológica (Delisle 1980), por lo que no se puede dejar de lado la enseñanza de lenguas extranjeras en la formación de traductores (Pym 1992).

Los estudios en didáctica de lenguas extranjeras en la formación de traductores e intérpretes son muy escasos. Sin embargo, tal y como apuntan López Ropero \& Tabuenca (2009: 124), "este estudio se hace todavía más necesario dado el modelo profesionalizador que fomenta el EEES". El sistema de competencias debería elaborarse en función de la realidad y exigencias del idioma dentro del mercado laboral, contribuyendo, de este modo, al desarrollo de la competencia traductora. Partiendo de esta base, el presente artículo tiene como objetivo principal la elaboración de un modelo de competencias específicas para la formación lingüística de futuros traductores e intérpretes a partir de un modelo de competencia traductora. Para ello, se establecerá, en primer lugar, un marco conceptual y tipológico de competencias y se llevará a cabo un análisis descriptivo sobre la enseñanza que aquí nos ocupa a partir 
de la bibliografía disponible hasta la fecha dentro de esta subdisciplina. En lo sucesivo, se presentará el modelo de competencia traductora del grupo PACTE, sobre el cual se basa la propuesta de competencias de este trabajo. Finalmente, se presentará el modelo de competencias propuesto.

\section{Hacia un marco de competencias: definición y tipología}

Según el Real Decreto 1393/2007 por el que se establece la Ordenación de las Enseñanzas Universitarias Oficiales en el estado español, los planes de estudio conducentes a la obtención de un título universitario deberán "tener en el centro de sus objetivos la adquisición de competencias por parte de los estudiantes". Con esta nueva normativa no solo se pretende poner el énfasis en los resultados de aprendizaje del estudiantado, sino también garantizar el acceso a un tipo de enseñanza contextualizada y funcional que no responda únicamente a la mera adquisición de conocimientos disciplinares (Pérez Gómez 2007). Así pues, la planificación de las enseñanzas debería desembocar en un aprendizaje deseado y centrado en los estudiantes que dé respuesta a las necesidades profesionales y académicas de un perfil previamente definido (Yániz 2006: 20; Bolívar 2007).

La necesidad de definir la educación superior a través de competencias responde a un proceso de reconstrucción social motivado por el proceso de globalización, las tecnologías de la información y la creciente multiculturalidad (Bolívar 2008). López Ruiz (2011: 291) se refiere a este cambio como un "genuino giro copernicano y de paradigma en la Educación Superior". Se trata de dar respuesta a unas necesidades actuales y, en la medida de lo posible, futuras que posibiliten a los estudiantes desarrollar amplias capacidades de aprendizaje y desaprendizaje a lo largo de toda su vida y, de este modo, adaptarse a situaciones cambiantes (Cano 2008). Para ello, será necesario dejar de lado la superespecialización y dar paso a un conocimiento integrado (Morin 2001). Aprender y hacer pasan a concebirse como acciones inseparables a través de una metodología basada en la resolución de problemas que permita emular situaciones auténticas o cercanas a la realidad (Bolívar 2007: 81). Tal y como apunta este mismo autor, este es el desafío al que se han enfrentado las universidades europeas, es decir, la transición de un modelo de enseñanza tradicional a otro basado en el aprendizaje autónomo del alumnado.

La enseñanza universitaria por competencias nace a partir del proyecto Sócrates-Erasmus titulado Tuning Educational Structures in Europe, el cual tiene como base la armonización y definición de las titulaciones universitarias en el proceso de convergencia educativa de Bolonia. En este proyecto el concepto de competencia se define como "una combinación de atributos que describen el 
nivel o grado de suficiencia con que una persona es capaz de desempeñarlos" (González \& Wagenaar 2003: 80). Para Cano (2008), una competencia implica: (a) integrar conocimientos; (b) realizar ejecuciones ligadas a la práctica; (c) actuar de forma contextual; (d) aprender constantemente; y (e) actuar de forma autónoma y con profesionalidad. Se trata, pues, de una nueva manera de entender la educación en la que el papel del profesor es ayudar a que los estudiantes adquieran una serie de habilidades vinculadas a su futuro profesional y les permitan seguir su proceso de aprendizaje a lo largo de sus vidas. El proyecto Tuning propone que los créditos europeos, o ECTS, que componen la estructura de cualquier titulación universitaria, se formulen en forma de competencias que determinen los logros de aprendizaje. Le Boterf (2000), por su parte, se refiere a una competencia como un saber combinatorio que el aprendiz construye a través de una secuenciación de actividades formativas. Así pues, una competencia se caracteriza por la aplicación de una manera práctica y efectiva de un conocimiento utilizable y de una serie de habilidades en un contexto específico (Bolívar 2008).

En las enseñanzas universitarias pueden distinguirse dos tipos de competencias. Por una parte, las competencias generales, las cuales son transversales y aplicables a cualquier titulación, a saber, la competencia comunicativa, la capacidad de liderazgo o la capacidad de trabajo autónomo, entre otras. Se trata de un conjunto de capacidades y destrezas que los alumnos pueden aplicar a cualquier ámbito de su vida. Por otra parte, las competencias específicas, las cuales son propias de cada titulación o área de estudio, tienen un fin profesionalizante. En el presente trabajo nos centraremos en este segundo tipo de competencias y, más concretamente, en aquellas aplicables a las asignaturas de Lengua B. Como ya se ha indicado, las competencias descritas en documentos oficiales referidas a la enseñanza de lenguas extranjeras para traductores e intérpretes en formación siguen siendo muy generales; de ahí la necesidad de proponer modelos de referencia que permitan al profesorado reorientar su docencia y moldearla según la nueva realidad educativa.

\section{Estudios previos: en busca de rasgos definitorios}

Hasta la fecha no se ha propuesto una matriz de competencias específicas que se aplique a las materias de lengua dentro de los Grados de Traducción e Interpretación. Sí existen, no obstante, propuestas de objetivos presentadas por otros autores. Tal y como apunta Bolívar (2008: 17), "el enfoque por competencias aboca a una planificación por objetivos de aprendizaje". Así pues, en este punto se analizarán las propuestas presentadas por distintos académicos. 
Debido a la falta de investigación, se analizarán estudios aplicados a la lengua B y C y a los idiomas inglés y alemán.

En lo que se refiere a las destrezas comunicativas, Berenguer (1997), pionera en este tipo de estudios, señala la necesidad de evitar una lectura mecánica de los textos, ya que estos forman parte de un ente global que adquiere sentido y significado por la suma de todos sus elementos. Como apunta Hurtado (2017: 3), "translation is not situated on the level of language but rather the level of parole". El énfasis debe recaer en el desarrollo de la comprensión lectora y análisis textual desde el punto de vista de la traducción, para lo cual será necesario centrar la atención en el desarrollo de la competencia discursiva. Los alumnos han de tener la capacidad de reconocer diferentes tipologías textuales, sus funciones, estructura y marcas textuales.

Brehm \& Hurtado (1999), en su propuesta de objetivos para la Lengua B Inglés, hacen una distinción de las destrezas atendiendo a la comprensión lectora, la expresión escrita y el uso oral. En la primera, afirman que el alumno debe adquirir distintas estrategias de lectura, a saber, estrategias de skimming y scanning y de lectura intensiva y extensiva. Asimismo, los alumnos deberán ser capaces de identificar, comprender y distinguir los mecanismos que contribuyen a la coherencia y cohesión del texto, el funcionamiento de distintos tipos y géneros textuales, las variaciones de registro o dialectales en los textos, los rasgos pragmáticos y semióticos del texto, las variaciones lingüísticas relacionas con el uso y el usuario y el desarrollo de un espíritu crítico hacia los textos. En el caso de la producción de textos escritos, estas autoras inciden en las estrategias de planificación textual como toma de notas o confección de esquemas previos, producción de textos de distintos tipos y géneros y de diversos campos, modos y tonos. Finalmente, para el uso oral, hacen hincapié en la capacidad de discernir distintos acentos (regionales y sociales), los distintos tipos y géneros de discurso oral, pronunciación y entonación y el desarrollo de estrategias comunicativas.

Möller (2001), en su propuesta de objetivos para la Lengua C Alemán, diferencia entre el desarrollo de la comprensión lectora y el resto de destrezas. La destreza de la comprensión lectora es la que mayor atención debería recibir, al menos durante los primeros años de la enseñanza del idioma. Esta autora hace hincapié, entre otros, en las formas de trabajar los textos. Se emplearán tanto métodos de lectura auténtica como analítica. En el primero se apelará a estrategias inductivas que permitan un estudio del texto de forma más superficial y, en el segundo, como su nombre bien indica, se llevará a cabo un análisis del texto más exhaustivo. Asimismo, en el análisis del texto se abordarán cuestiones contextuales e intratextuales. Finalmente, esta autora propone que 
el resto de destrezas se trabajen de forma autónoma debido al escaso tiempo disponible en la enseñanza de la Lengua C. ${ }^{2}$ Se propone que la comprensión oral se trabaje a partir de material audiovisual y la expresión oral a través de intercambios comunicativos con otro interlocutor.

Clouet (2010) incluye siete destrezas comunicativas en el caso de la Lengua B Inglés, a saber, expresión oral, expresión escrita, comprensión auditiva, comprensión lectora, comprensión audiovisual, interacción oral e interacción escrita. Al igual que en las propuestas anteriores, estas habilidades tienen como finalidad el desarrollo de la competencia discursiva o textual a partir de diferentes géneros orales y escritos, incluyendo tanto procedimientos analíticos como de producción. Todo esto debe suscitar la reflexión sobre el funcionamiento de la lengua inglesa en sus niveles morfosintácticos, léxicos y fonológicos, así como su transcendencia comunicativa en ámbitos generales, semiespecializados y especializados.

\subsection{La contrastividad lingüistica como elemento central}

La investigación realizada hasta la fecha coincide en la necesidad de estudiar la lengua desde la contrastividad (Berenguer 1997; Brehm \& Hurtado 1999; Möller 2001; Clouet 2010; Cerezo 2013, 2015). Debido al hecho de que un traductor trabaja de forma paralela con dos códigos lingüísticos, resulta imprescindible estudiar ambas lenguas en contacto desde una perspectiva contrastiva. Berenguer (1997) insiste en que este análisis contrastivo debería abordar la contrastividad de una manera dinámica a partir del texto. No debe entenderse, pues, como una comparación estática de reglas y sistemas gramaticales, es decir, no debe limitarse a confrontar dos sistemas lingüísticos. Esta debe basarse, más bien, en el contraste de ambas lenguas en situaciones comunicativas concretas. Brehm y Hurtado (1999) aplican este enfoque contrastivo, por una parte, al plano léxico: falsos amigos, juegos de palabras, expresiones idiomáticas, abreviaturas y siglas de uso frecuente, terminología de campos de especialidad, sinónimos y tratamiento de inequivalencias en la lengua materna y, por otra, al plano gramatical: calcos, estructuras sin equivalencia en la lengua materna, mecanismos de coherencia y cohesión y conocimiento de las convenciones propias de los lenguajes de especialidad.

2. Hay que tener en cuenta que la mayoría de facultades de Traducción e Interpretación en España no exigen conocimientos previos en la Lengua C, aunque sí es necesario que desarrollen una competencia suficiente en el transcurso de dos años como para poder llevar a cabo traducciones directas desde esta lengua. 
Möller (2001), por su parte, explica que la contrastividad debería entenderse más bien como un enfoque de la enseñanza de la lengua y no como un objetivo en sí. Dicha contrastividad debe darse, siguiendo la distinción saussureana, en el nivel de la parole y no de la langue. Esta autora explica, además, que el uso de la lengua materna no debería estar reñido con la enseñanza de la lengua extranjera, ya que la traducción es el fin al que nos dirigimos. Ahora bien, este uso de la Ll no debería determinar la metodología del curso ni eximir el uso activo de la lengua extranjera en el aula.

A diferencia de las propuestas anteriores, Clouet (2010) propone un uso intermitente de un enfoque contrastivo. La competencia contrastiva debe concienciar a los estudiantes de que cada lengua cuenta con estructuras propias. El estudio contrastivo, pues, debe partir del análisis de dificultades y diferencias que tengan una clara influencia sobre las tareas de mediación. Para ello, además del plano léxico al que aludían Brehm \& Hurtado (1999), la contrastividad también debe aplicarse al plano morfosintáctico (los verbos modales, el uso de preposiciones, tiempos verbales, adjetivos, etc.) y al plano textual (conectores, marcadores textuales, mecanismos de coherencia y cohesión textual, etc.).

\subsection{El enfoque: enseñanza de lenguas con fines específicos}

La formación en lenguas en las titulaciones de Traducción e Interpretación debe partir de la enseñanza de lenguas extranjeras con fines específicos, buscando, de ese modo, un diseño curricular basado en el análisis de necesidades específicas de los discentes. Tal y como apunta Berenguer (1997: 30), este enfoque "prioritza l'aspecte comunicatiu de la llengua [...] i diferencia els objectius d'aprenentatge en funció de l'ús que en farà l'estudiant en el món professional". Almagro (2001) también defiende que, a diferencia de la enseñanza general de la lengua, este enfoque despierta la motivación del alumno al entender que la formación responde a una necesidad profesional. El estudiante percibe una relación entre aquello que estudia y su futura profesión (Oster 2008). Asimismo, permite centrar la atención en una o determinadas destrezas, lo cual posibilita graduar el tratamiento que estas reciben a través de las distintas asignaturas (en primer lugar, las destrezas escritas y, posteriormente, las orales). En la enseñanza general de lenguas, las distintas destrezas comunicativas suelen estar presentes en el aula desde los niveles básicos hasta los avanzados y se suelen trabajar todas por igual.

Por otra parte, este enfoque permite introducir en mayor medida materiales auténticos en el aula, a saber, textos periodísticos, especializados, ponencias, etc., y acotar la formación a la tipología de textos que los estudiantes traducirán en el futuro con mayor frecuencia. Cruz \& Mulligan (2004) inciden en que, 
desde el momento inicial, los estudiantes han de ser expuestos a una amplia gama de tipologías textuales que les permita afrontar con mayores garantías de éxito las posteriores asignaturas de traducción e interpretación y conseguir una integración más rápida y efectiva en el mercado laboral (Cerezo 2015).

\subsection{Otros elementos clave}

El conocimiento cultural es otro de los puntos que debe caracterizar esta enseñanza. La lengua y la cultura van unidas, por lo que carece de sentido disociar una de la otra. En este sentido, Clouet (2010) incide en que este conocimiento cultural implica el desarrollo de convenciones lingüísticas como los registros, los dialectos, la interpretación de referentes culturales y el análisis connotativo, así como el análisis de la carga cultural de entidades semióticas, determinadas unidades léxicas, aspectos morfosintácticos y géneros textuales.

Otro aspecto muy señalado en las distintas propuestas es el uso de fuentes de documentación. Resulta fundamental entrenar y desarrollar la labor de documentación previa a la traducción (Soriano 2004). Para ello, hay que familiarizar al alumno con diccionarios no solo bilingües (Möller 2001), sino también monolingües, gramáticas, enciclopedias, manuales de estilo, diccionarios especializados, diccionarios de sinónimos y antónimos, de frases hechas, etimológicos, de argot, etc. (Berenguer 1997). El alumno debe desarrollar, además, la capacidad de extrapolar la información pertinente de las definiciones, elegir la acepción adecuada o saber a qué fuente o fuentes de referencia debe recurrir (Brehm \& Hurtado 1999).

Otro punto común es el desarrollo de la autonomía del alumno (Möller 2001; Soriano 2004; Clouet 2010; Cerezo 2013). El mercado laboral actual se caracteriza por su volatilidad, por lo que la universidad no puede proveer al alumno de todos los conocimientos existentes. Esto nos lleva a incorporar la competencia aprender a aprender, al igual que en el MCER (Marco Común Europeo de Referencia para las Lenguas), como un pilar fundamental de esta enseñanza. En esta línea, Clouet (2010) también se refiere a la competencia estratégica como una macrocompetencia que orquestra todas las demás. Se trata de estrategias de carácter metacognitivo, cognitivo, afectivo y social que deben favorecer el fomento de las destrezas comunicativas. Soriano (2004) también menciona como aspectos de especial relevancia el trabajo en equipo y consultas a especialistas, entrenar el espíritu crítico, la autoconfianza y participación activa en el proceso de aprendizaje, la capacidad de organización y resolución de problemas, la evaluación, la autoevaluación y la toma de decisiones. 
Finalmente, en algunas propuestas también se habla de sensibilizar al alumno con la actividad traductora. Se trata de comentar aspectos relacionados con el proceso traductor y diferentes modelos teóricos, problemas de traducción, crítica de traducciones, aspectos relacionados con la práctica profesional y los estudios y crear situaciones profesionales reales en el aula (Berenguer 1997). Möller (2001) destaca que no se trata de que el alumno aprenda a traducir en la clase de lengua, sino de que entienda la lengua extranjera desde la suya propia a partir de fenómenos culturales, lingüísticos, etc.

\section{Un modelo de competencia traductora}

Partimos de la base de que cualquier propuesta de competencias para la enseñanza de la lengua extranjera en una titulación de Traducción e Interpretación debe elaborarse teniendo en cuenta el objetivo final de la titulación, es decir, el desarrollo de la competencia traductora (en lo sucesivo, CT). El grupo PACTE (2011: 318) define la CT como "the underlying system of knowledge required to translate". ${ }^{3}$ Tal y como apunta Oster (2008: 4), "la didáctica de las lenguas para traductores necesita el referente de la traducción como actividad profesional para poder determinar sus objetivos y también su metodología". Stalmach (2009: 95), en una línea similar, afirma que los contenidos, objetivos y niveles de enseñanza deben basarse en: (a) los estudios y la didáctica; (b) el contenido de las clases de traducción; y (c) el mercado laboral.

Así pues, a raíz de estas consideraciones previas, nuestra propuesta se basará en el modelo holístico de CT propuesto por el grupo PACTE, ya que se trata de un modelo que, a diferencia de otros, ha sido sometido a una investigación de tipo empírico-experimental y, como consecuencia de ello, se presenta un modelo actualizado según los resultados obtenidos en dicha investigación. Ahora bien, cabe destacar que, incluso desde antes del nacimiento de la Traductología como ciencia en la década de los años 80, las propuestas de modelo de CT han sido notables (Wilss 1976; Delisle 1980; Roberts 1984; Hewson \& Martin 1991; Neubert 1994: Kiraly 1995; Hansen 1997; Risku 1998; Kelly 2002).

PACTE (2017) incorpora las siguientes cinco subcompetencias a su modelo de CT:

3. PACTE (Proceso de Adquisición de la Competencia Traductora y su Evaluación). Grupo de investigación de la Universitat Autònoma de Barcelona. 


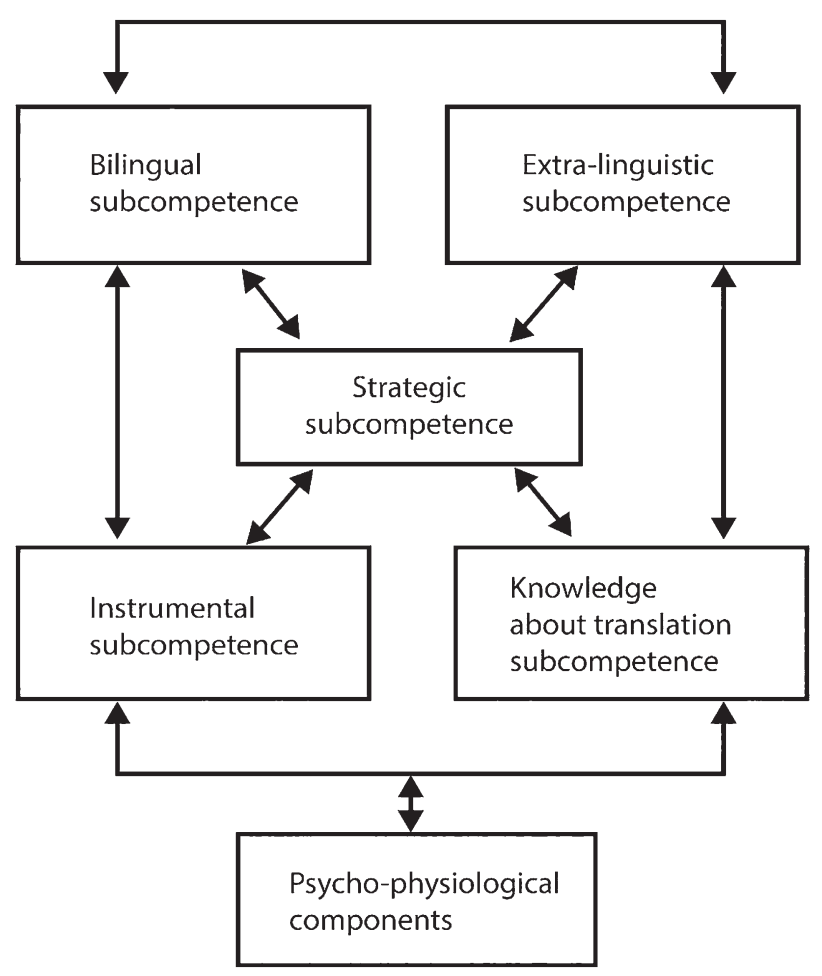

Fig. 1. Modelo de Competencia Traductora (PACTE 2017)

- Subcompetencia bilingüe: conocimiento mayoritariamente procedimental necesario para la comunicación en dos lenguas. Este conocimiento incluye aspectos pragmáticos, sociolingüísticos, textuales, gramaticales y léxicos.

- Subcompetencia extralingüística: conocimiento mayoritariamente declarativo, implícito y explícito sobre el mundo en general, así como especializado. Incluye un conocimiento bicultural, enciclopédico y temático.

- Subcompetencia de conocimiento en traducción: conocimiento mayoritariamente declarativo, implícito y explícito sobre la traducción y sus aspectos profesionales. 
- Subcompetencia instrumental: conocimiento mayoritariamente procedimental relacionado con el uso de fuentes de documentación, información y tecnologías de la comunicación aplicadas a la traducción.

- Subcompetencia estratégica: conocimiento de tipo procedimental que garantiza la eficacia del proceso traductor. Se trata de una subcompetencia esencial que afecta a todas las demás. Esta subcompetencia permite compensar posibles deficiencias, identificar problemas de traducción y aplicar soluciones. Además, actuará según la direccionalidad de la traducción (traducción directa o inversa), la combinación lingüística, la especialización (textos técnicos, legales, audiovisuales, etc.), la experiencia del traductor y el contexto de la traducción.

Además de estas cinco subcompetencias, PACTE también establece una serie de componentes psicofisiológicos, es decir, diferentes tipos de componentes cognitivos y actitudinales que determinan el proceso traductor, a saber, la memoria, la atención, la curiosidad intelectual, la perseverancia, etc. En estos componentes también se incluyen los mecanismos psicomotores.

\subsection{Consideraciones previas}

El modelo de CT presentado por el grupo PACTE será utilizado en este trabajo como base para la construcción de un modelo de competencias que defina las asignaturas de Lengua B conforme a las nuevas directrices europeas para la educación superior. Sin embargo, teniendo en cuenta la naturaleza de la enseñanza que aquí nos ocupa, estimamos necesario rediseñar el modelo presentado, ya que el papel que juegan las distintas subcompetencias en las materias lingüísticas es cualitativamente distinto al de las propias asignaturas de traducción. En las asignaturas de lengua, el objetivo principal es preparar lingüísticamente al alumno para afrontar las distintas asignaturas de traducción e interpretación que componen los planes de estudio, mientras que las asignaturas de traducción e interpretación buscan dotar al alumno de estrategias que le permitan trasladar el material textual de una lengua a otra. Así pues, proponemos la siguiente redistribución del modelo de CT del grupo PACTE: 


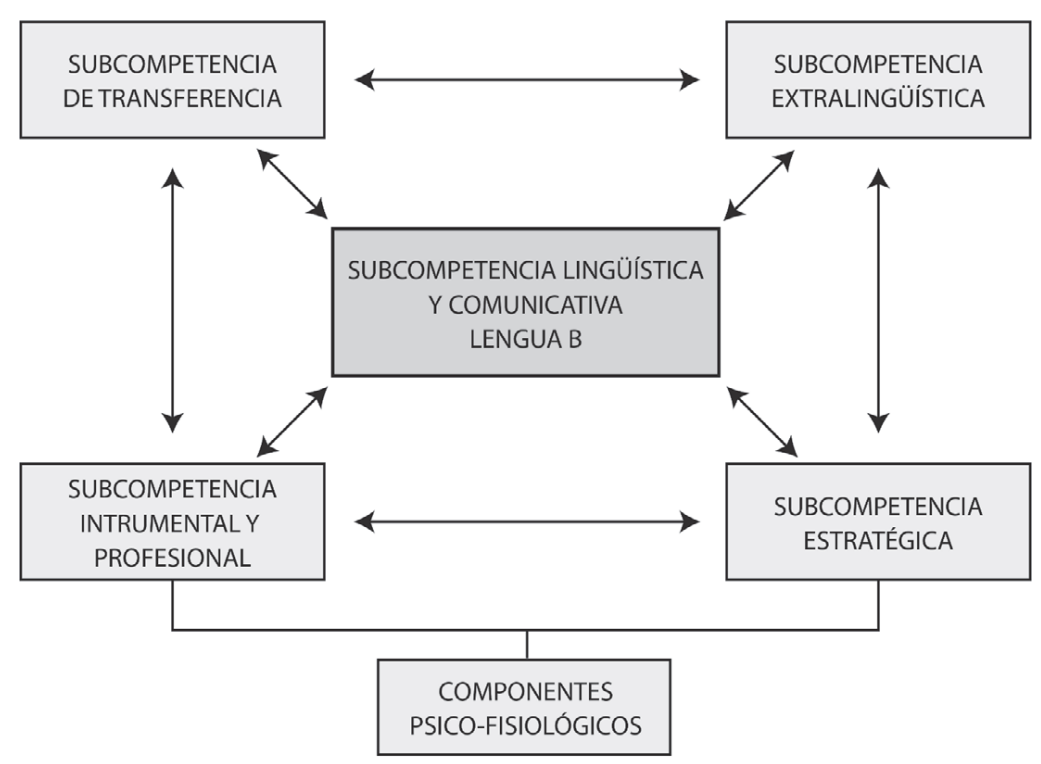

Fig. 2. Reorganización del modelo de CT para Lengua B

Pese a que no es este el espacio para un análisis exhaustivo del modelo presentado por PACTE, llama la atención que en él se habla de una subcompetencia bilingüe, lo cual supone un cambio con respecto a la denominación que esta subcompetencia recibía en su modelo inicial de 1998. El bilingüismo, pese a ser deseable para la traducción profesional, no es un requisito sine qua non. Tal y como apunta Hurtado (1999b), la condición de bilingüe no garantiza el éxito profesional de un traductor por ser la CT cualitativamente distinta a la competencia bilingüe (PACTE 2017). Presas (2003), en este sentido, apunta hacia un problema de definición conceptual. Aunque existen estudiosos que tradicionalmente han atribuido la etiqueta de bilingüe a aquellos sujetos con una capacidad nativa o casi nativa en dos lenguas (Bloomfield 1933), con el paso del tiempo la investigación apunta hacia una definición basada en la capacidad de uso de dos códigos lingüísticos de forma alternativa (Mackey 1970; Titone 1972; Lam 2001; Grosjean 2010). Por eso, en este trabajo esta subcompetencia se etiquetará como subcompetencia lingüística y comunicativa y se ubicará en el centro de todo el proceso.

Por otra parte, también estimamos necesario incluir una subcompetencia de transferencia. Hurtado (1999a: 44) enfatiza que "la competencia de transferencia es crucial, ya que en ella se integran todas las demás". Colina (2002) afirma que muchos estudiantes de traducción, pese a poseer una amplia 
competencia en la lengua extranjera, tienen problemas a la hora de realizar actividades de traducción por una falta de control de transferencia. De hecho, Neubert $(1994,2000)$ defiende que esta competencia es la que caracteriza y distingue a un traductor de, por ejemplo, escritores u oradores en su lengua materna, estudiantes de segundas lenguas o lenguas extranjeras, expertos en diversas ramas del conocimiento, etc. Wills (1982) se refiere a esta capacidad de transferencia como una super competencia que posibilita la mediación entre la L1 y la L2. Al fin y al cabo, la habilidad de un traductor radica en no confundir y mezclar dos lenguas en contacto. Siguiendo a Duff (1981), un claro ejemplo en inglés sería hard eggs (traducción directa del español) en lugar de boiled eggs. Esta capacidad es, de hecho, un indicador del conocimiento de esas lenguas y su aplicación profesional (Delisle 1988: 21). En el modelo inicial de PACTE, la subcompetencia de transferencia ocupaba el lugar central, siendo reemplazada por la subcompetencia estratégica en su modelo definitivo.

Ahora bien, es digno de mención que dicha experimentación empírica se llevó a cabo con un grupo de traductores profesionales que, por la naturaleza de la propia profesión, ya había desarrollado previamente la competencia de transferencia. También se contó con un grupo de profesores de lengua extranjera, quienes de forma indirecta habían desarrollado una habilidad traductora natural (PACTE 2017) o innata (Harris \& Sherwood 1978) por la inevitable comparación de la L1 y L2 que se produce durante el proceso de aprendizaje de la segunda. PACTE distingue entre un estudio de la competencia traductora per se y la adquisición de la CT en estudiantes de traducción. Esto, pues, justifica nuestra necesidad de adaptar el modelo de CT a la realidad del aula, sobre todo en los primeros cursos de la titulación, momento en que se integran las asignaturas de contenido lingüístico y los estudiantes todavía no han tenido un contacto previo con la propia actividad traductora.

Finalmente, se ha decidido unificar la subcompetencia instrumental y de conocimiento en traducción por ser subcompetencias que se desarrollarán más profundamente en las asignaturas de traducción.

\section{Propuesta de competencias para las asignaturas de Lengua B}

Presentamos a continuación nuestra propuesta de subcompetencias atendiendo al desarrollo de una competencia madre, la competencia traductora. Partimos de la premisa de que el estudiante del Grado de Traducción e Interpretación ya goza de cierto nivel lingüístico en su primera lengua extranjera a la hora de acceder a los estudios. Por esta razón, en la línea de Brehm \& Hurtado (1999), las competencias planteadas tendrán como objetivo principal consolidar 
aquellos conocimientos que ya se poseen y adquirir otros nuevos a nivel más avanzado.

$\rightarrow$ SP1: Subcompetencia lingüística y comunicativa (Lengua B)

Fomento de las cuatro destrezas comunicativas, a saber, comprensión y expresión orales y escritas atendiendo a las exigencias lingüísticas impuestas por la naturaleza de la propia actividad traductora.

En líneas generales, la subcompetencia lingüística debe estar al servicio de la subcompetencia comunicativa (Clouet 2010), ya que la lengua no es un fin en sí mismo, como ocurre en las titulaciones de Filología, sino un medio para conseguir otro fin, es decir, trasladar el material textual de una lengua a otra (Berenguer 1997). Así pues, el alumnado ha de tener la capacidad de comprender a la perfección textos orales y escritos especializados y no especializados de diversa índole, así como de redactar textos tanto de carácter general como semiespecializados, en este segundo caso con la ayuda de textos paralelos. También han de desarrollar la capacidad de hablar en público de forma espontánea sobre temas generales y sobre temas específicos con una preparación previa.

La comprensión escrita debería caracterizarse por la capacidad de desverbalizar los textos y de entender los significados tanto implícitos como explícitos ${ }^{4}$ a través de diferentes estrategias de lectura. Para el desarrollo de esta capacidad de desverbalización, el estudio de la lengua se hará desde un punto de vista descriptivo, sobre todo a nivel léxico-semántico, y se fomentará la capacidad de deducción de palabras desconocidas en contexto. Además, también se desarrollarán estrategias de análisis discursivo y textual aplicadas a la traducción (Berenguer 1997), así como de pre-traducción pedagógica de frases o fragmentos cortos contextualizados. Por otra parte, se debe poner el énfasis en la comprensión oral a través de ejercicios pedagógicos que desarrollen la escucha activa del alumnado (Bowen $\&$ Bowen 1984: 6) y que garanticen un alto nivel de concentración, así como una comprensión completa del mensaje trasmitido (Cerezo 2013, 2017). Para ello podrán llevarse a cabo ejercicios tanto intralingüísticos como interlingüísticos que permitan el predesarrollo de las habilidades cognitivas que subyacen al proceso de interpretación como, por ejemplo, repartir la atención, trabajo simultáneo con dos códigos lingüísticos, análisis discursivo, uso de la memoria a corto plazo, etc. La implementación de estas capacidades será gradual. En este sentido, López Ropero y Tabuenca (2009) proponen que, en primer lugar, se desarrolle la memoria a corto plazo

4. Desverbalización: término acuñado por Seleskovitch \& Lederer (1984). 
$y$, posteriormente, la toma de notas, ya que el fin de las notas es complementar la memoria de un intérprete, y no a la inversa.

En la expresión escrita se abordará la producción de textos de diversa índole teniendo en cuenta las tendencias del mercado laboral y aquellas disciplinas que requieran una amplia demanda de traducción inversa (Mayoral 2001). En el caso de la producción oral se promoverán, por una parte, ejercicios de perfeccionamiento fonético y, por otra, ejercicios que desarrollen la competencia oral comunicativa. Así pues, se desarrollará tanto la fluidez (fluency) como la precisión (accuracy), y se hará hincapié en aquellos aspectos que presenten más problemas para los discentes. Además, con el fin de conseguir un aprendizaje autónomo y continuo de cara al futuro, se introducirá la lectura de transcripciones fonéticas.

En líneas generales, siguiendo el enfoque socio-constructivista de Kiraly (2000), se trata de que a través del desarrollo de las destrezas lingüísticas los estudiantes consigan un aprendizaje efectivo de la lengua como paso previo a la traducción. Oster (2008), tomando como referencia la dicotomía adquisiciónaprendizaje propuesta por Krashen (1982), afirma que el énfasis debería recaer en la adquisición de la lengua por presentar este proceso un fuerte componente somático. Lo que debe buscarse es principalmente el desarrollo de una competencia comunicativa basada en una confianza comunicativa. Además, Stalmach (2008: 100) aboga por un whole language approach, donde las distintas destrezas se estudien en conexión con las otras.

Desarrollo de los principales elementos lingüisticos que intervienen en el proceso de traducción e interpretación

Un traductor debe tener la capacidad de entender la relación existente entre los distintos elementos macrotextuales y microtextuales que componen un texto. Para ello se hará hincapié, por una parte, en las convenciones textuales del texto y, por otra, en los mecanismos de organización textual que contribuyan a un entendimiento del texto como ente global (Berenguer 1997).

En este punto cabe destacar los siguientes niveles lingüísticos:

- Mecanismos de coherencia y cohesión que dan forma a la progresión temática del texto.

- Acentos, dialectos geográficos y sociales e idiolectos. Se trata de aspectos fundamentales para aquellos estudiantes que quieran dedicar su carrera profesional a la interpretación, bien sea de conferencias o de enlace. La dialectología debería ser tal vez el aspecto más importante dentro de esta categoría por presentar habitualmente las lenguas una amplia 
variedad de acentos que pueden llegar a dificultar en gran medida la comprensión de un discurso.

- Diferentes registros lingüísticos. Por lo general, el registro vendrá determinado por el tipo de traducción en sí. En un artículo científico, el lenguaje será culto, con gran cantidad de oraciones subordinadas, presencia de latinismos, etc. Sin embargo, en un texto audiovisual, el registro será más coloquial y propio del lenguaje oral. Así pues, se abordarán ambos tipos.

- Estructuras gramaticales que dan forma al texto.

- Rasgos pragmáticos y semióticos del texto, especialmente aquellos elementos que puedan tener un efecto sobre el significado, a saber, los elementos intertextuales, connotativos, las implicaturas, etc.

- Variaciones textuales y de registro en relación al campo, modo y tono del discurso.

Conocimiento de los géneros textuales escritos y orales más recurrentes y sus convenciones

Un traductor trabaja con textos. Un amplio conocimiento de tipologías y convenciones textuales puede ayudarle a agilizar el proceso de traslación y adoptar determinadas soluciones de traducción. En este sentido, Cruz \& Mulligan (2004) abogan por la introducción de una amplia variedad de géneros textuales desde el inicio de la formación, siendo las asignaturas de lengua extranjera las primeras que deben hacerse eco de esta necesidad formativa. Se hará hincapié sobre todo en aquellas tipologías textuales pertenecientes a disciplinas que más suelen traducirse, a saber, traducción jurídica y jurada, científico-técnica, etc. Mediante este enfoque de géneros textuales, también se busca que el alumno entienda que la traducción es una operación textual (Hurtado 2011). Todos y cada uno de los elementos que componen un texto cobran sentido cuando se integran en su totalidad, y de ahí la necesidad de desarrollar un conocimiento profundo de las distintas tipologías textales y sus convenciones. Así, además de ser capaces de reconocerlas y aplicarlas, también es necesario que anticipen aspectos como posibles subtipos y la organización discursiva.

Adquisición de un vocabulario general amplio y terminología especializada Un texto adquiere sentido a través de las palabras, por lo que un traductor debe poseer un profundo conocimiento léxico. El énfasis debe recaer en el estudio semántico de las palabras, con especial énfasis en su connotación y registro. También conviene familiarizar al estudiante con determinadas rutinas 
lingüísticas que atiendan a distintas situaciones comunicativas (Hatim \& Mason 1990: 48). En cuanto a la terminología especializada, se incluirá el lenguaje jurídico, económico, científico y tecnológico. Este conocimiento otorgará a los estudiantes una mayor fluidez en el proceso de traslación y les permitirá descargar su capacidad cognitiva, la cual ha de atender a distintos procesos mentales paralelos que subyacen en el propio proceso de traducción.

\section{$\rightarrow$ SP2: Subcompetencia de transferencia}

Desarrollo de la agilidad de reconocimiento de estructuras y unidades léxicas

Como ya hemos indicado, un traductor, a diferencia de una persona competente en dos lenguas, debe tener la capacidad de distinguir y separar ambas lenguas en contacto (PACTE 2017). Dado que en su labor diaria un traductor contrapone dos sistemas lingüísticos que difieren entre sí, resulta pertinente que en las asignaturas de lengua extranjera se resalten las principales diferencias entre ellas. De este modo se contribuye a que en las posteriores asignaturas de traducción e interpretación disminuyan los errores originados por el contacto entre ambas. De igual manera, mediante esta competencia se pretende fomentar la fluidez en el proceso de traslación. Berenguer (1997) y Oster (2008) inciden en la necesidad de sacar el mayor provecho posible a esta subcompetencia. Para ello, no se debe limitar su aplicación a una pura contraposición de niveles lingüísticos, sino que este análisis contrastivo debería abarcar desde el nivel fonológico hasta el nivel textual y el estilo. Además, conviene aprovechar las similitudes para facilitar el aprendizaje.

Teniendo todo esto en cuenta, dentro de esta categoría incluimos los siguientes puntos:

- Conocimiento profundo de aspectos gramaticales de la lengua, sobre todo aquellos que por su lejanía difieran con la Ll del estudiante. Se deben contraponer ambos sistemas gramaticales para que el alumno pueda ver y entender las diferencias entre ellas. Este conocimiento sobre el sistema gramatical de la lengua debe servir también para crear mecanismos de anticipación a través de deducciones basadas en estructuras sintácticas, sobre todo para la interpretación.

- Conocimiento profundo de falsos cognados.

- Reconocimiento de ironías, juegos de palabras e inequivalencias léxicas con la Ll del estudiante.

- Utilización correcta y apropiada de los signos de puntuación. 
- Conocimiento y apreciación de las diferencias de estilo entre ambas lenguas.

- Pronunciación correcta e inteligible de sonidos, sobre todo aquellos que no existen en la lengua materna del estudiante, como algunos sonidos vocálicos en inglés para un hispanohablante, los cuales pueden llegar a ser una verdadera barrera de comunicación.

Esta subcompetencia no debe introducir la traducción como metodología de aprendizaje, ya que la enseñanza dejaría de tener un enfoque comunicativo. El objetivo es más bien centrar la atención en aspectos concretos que pueden resultar importantes para su futuro profesional.

$\rightarrow$ SP3: Subcompetencia extralingüística

Adquisición de conocimientos culturales de los principales países donde el idioma objeto de estudio sea oficial

El estudio de la lengua no debe desligarse en ningún caso del conocimiento cultural de los países donde esta sea oficial. Las lenguas expresan realidades propias del contexto donde estas tienen lugar, por lo que el aprendizaje no puede ser auténtico si esta realidad no se tiene en consideración (Peterson y Coltrane 2003). De hecho, la investigación en Traductología apunta en una misma línea. Snell Hornby (1988) considera la traducción como un "acto transcultural", idea en la que también inciden Hewson \& Martin (1991) al referirse a esta como una "ecuación cultural" y al traductor como un "operador cultural". Reiss \& Vermeer (1991) prefieren hablar de "translación cultural" y no de "translación lingüística". Hatim \& Mason (1990) también entienden la traducción como un acto de comunicación que tiene lugar en un lugar concreto.

Esta subcompetencia, por las restricciones temporales de los cursos de lengua, debe centrarse en los "elementos que plantean problemas al alumno que no puede solucionar ni con una gramática ni con un simple diccionario" (Clouet \& Wood 2007: 107). Para ello deben abordarse aspectos que conforman principalmente la estructura sociedad actual como el sistema político y jurídico, el sistema educativo, creencias, cuestiones geográficas, tradiciones, fiestas, ocio, prensa, televisión, costumbres, música, religiones, arte, etc. Del mismo modo, también se incidirá en aspectos históricos que determinen patrones actuales de conducta social. Todo ello debe contribuir a la apreciación de la diversidad y la interculturalidad.

Esta subcompetencia también debe incluir el conocimiento de frases hechas, proverbios, colocaciones y estilística (Berenguer 1997). Se trata de 
elementos muy arraigados en el acervo cultural de cada lengua. El conocimiento de este tipo de frases redundará en una mayor seguridad y fluidez en el uso de la misma y permitirá, además, que el alumno con un nivel de lengua avanzado se aproxime a la competencia de un hablante nativo.

Adquisición de un conocimiento enciclopédico relativo a distintas áreas y especialidades, sobre todo aquellas que son más susceptibles de ser traducidas

Berenguer (1997) hace referencia a la heterogeneidad temática que caracteriza a un traductor. Las asignaturas de Lengua B deben constituir un espacio de aprendizaje no solo lingüístico, sino también enciclopédico a través de proyectos y trabajos grupales. Mackenzie (1998) también incide en la necesidad de combinar el conocimiento lingüístico con el conocimiento general, ya que un traductor ha de estar preparado para afrontar textos de diversa temática y con distintas necesidades comunicativas en su trabajo. El conocimiento enciclopédico permite con frecuencia entender un contenido más allá del mero significado de las palabras, lo cual es fundamental para que un traductor pueda desempeñar su labor con garantías de éxito. De ahí que se deba fomentar la lectura de textos específicos con el fin de que no solo aporten a los discentes conocimientos sobre la lengua, sino que también pueda suplir posibles lagunas de conocimiento general.

$\rightarrow$ SP4: Subcompetencia estratégica

Capacidad de afrontar problemas lingüísticos y de compensación de destrezas comunicativas

Como se ha explicado anteriormente, esta subcompetencia es la central en el modelo actual y definitivo del grupo PACTE. Se trata de una subcompetencia fundamental que controla todas las demás. En la adaptación del modelo de PACTE al tipo de formación que aquí nos ocupa, esta subcompetencia ha perdido su ubicación central para ceder el paso a la subcompetencia lingüística y comunicativa, pero ello no resta valor a su papel dentro de esta enseñanza.

Mediante esta subcompetencia se espera que se desarrollen técnicas individuales que ayuden al estudiantado a afrontar los problemas que se les presentan en los diversos textos y que derivan directamente del material lingüístico. Además, deberán desarrollarse mecanismos de compensación entre destrezas según se trate de traducción o interpretación y la direccionalidad (directa o inversa) (Hurtado 2017). Cada vez más, el mercado laboral de la traducción e interpretación exige que sus profesionales sean versátiles y que puedan enfrentarse a cualquier tipo de texto y temática. Existen numerosas variantes 
de la traducción, casi tantas como aventureros de la misma (Fuentes 2001). Además, el mercado laboral no distingue entre lenguas (A, B o C). A los ojos de la sociedad, los traductores son profesionales con una capacidad simétrica en sus lenguas de trabajo (Beeby 1996), por lo que no se espera que tengan problemas de direccionalidad a la hora de traducir (Clouet 2008). Asimismo, se espera que puedan abordar cualquier disciplina, por especializada que esta sea. Ante un mercado tan exigente, esta subcompetencia adquiere un valor especial. Los estudiantes han de saber equilibrar todas las subcompetencias aun cuando exista un déficit de conocimiento en alguna destreza lingüística y han de poseer los recursos necesarios para afrontar situaciones en las que se produzca un desequilibrio.

$\rightarrow$ SP5: Subcompetencia instrumental y profesional

Capacidad de aprender a manejarse con diversos materiales de consulta

Esta subcompetencia debe asegurar el acceso de los estudiantes a materiales de consulta de diversa índole y permitir un equilibrio con el resto de subcompetencias. Es importante concienciar a los futuros traductores de que la documentación en la lengua extranjera debe ocupar una parte importante del proceso de traducción. Los diccionarios, por razones obvias, son los que acompañan a un traductor la mayor parte del tiempo (Vinay \& Darbelnet 1970). Sin embargo, siguiendo a Delisle (1993) y Brehm (2007), debe evitarse el uso exclusivo de diccionarios bilingües por las limitaciones que estos presentan para la traducción. Deben utilizarse también diccionarios monolingües, de sinónimos y antónimos, gramáticas, etc. En este sentido, será especialmente relevante la instrucción en la elaboración de dosieres para así archivar vocabulario trabajado en el aula y que pueda servir como material de consulta en el futuro.

Ahora bien, Brehm \& Hurtado (1999) subrayan la necesidad de no depender de diccionarios y emplear técnicas de derivación de significado. Esta subcompetencia debe enfocarse hacia el desarrollo del trabajo de investigación con el objeto de enriquecer la producción de los discentes en la lengua extranjera. Para ello, Andreu et al. (2002) proponen el uso de una metodología basada en tareas que requieran un proceso de documentación previo. 


\section{$\rightarrow$ SP6: Componentes psico-fisiológicos}

Desarrollo de capacidades personales que resulten útiles para el ejercicio profesional de la traducción e interpretación

El fin de esta subcompetencia, en cuanto a las asignaturas de lengua se refiere, debe radicar en el desarrollo de la competencia empática, es decir, ejercitar ciertas actitudes y facultades cognitivas que favorezcan la comprensión e interpretación de un texto desde el punto de vista de la traducción (Andreu et al. 2002). Entre ellas, cabe destacar las siguientes:

- Habilidades cognitivas: aplicación práctica del conocimiento, adaptación a nuevas situaciones, reflejos, creatividad, crítica y autocrítica, trabajo autónomo e investigación.

- Capacidad de análisis y síntesis.

- Razonamiento lógico, deductivo e inductivo.

- Capacidad de iniciativa, seguridad en sí mismos y toma de decisiones.

También se introducirán ciertas competencias transversales, como las habilidades sociales, a saber, habilidades interpersonales, liderazgo, trabajo en equipo o trabajo interdisciplinar. También se fomentará, de acuerdo con las directrices europeas en materia educativa, la competencia aprender a aprender para asegurar la continuidad en la adquisición de conocimientos tras el paso por la universidad y el aprendizaje a partir de errores.

\section{Conclusiones}

La planificación por competencias en la enseñanza de lenguas extranjeras en la formación de traductores e intérpretes requiere atención e investigación urgente. Suscribimos la propuesta del grupo GRELT ${ }^{5}$ sobre la creación de una red de profesores que permita llevar a cabo una implantación correcta y adecuada del modelo de enseñanza que aquí nos ocupa. Dicha red de colaboración debería centrarse, entre otros, en el desarrollo óptimo de competencias para cumplir con las directrices europeas en materia educativa. De este modo se evitaría, tal y como apunta Bolívar $(2007,2008)$, que esta nueva realidad educativa por competencias se quede en un mero discurso que imposibilite a los estudiantes acceder a una enseñanza de carácter funcional diseñada a imagen y semejanza de su futura actividad profesional. Para ello, será necesario que,

5. Grup de Recerca en l'Ensenyament de Llengües Estrangeres per a Traductors de la Universitat Autònoma de Barcelona. 
por una parte, el alumnado deje atrás su papel pasivo y adopte una actitud activa en el proceso de aprendizaje (Imbernon \& Medina 2005; Bolívar 2007; Prieto 2008) y, por otra, que el profesorado evite su rol de instructor a través de clases magistrales y adopte un papel de facilitador de aprendizaje (Cano 2008).

La propuesta de competencias presentada en este trabajo se construye sobre dos pilares fundamentales: por una parte, mejorar el proceso de formación lingüística de los alumnos de Traducción e Interpretación a partir de las directrices del EEES y, por otra, dotar al alumnado de todos aquellos elementos lingüístico-culturales necesarios que contribuyan a su éxito profesional. Cano (2008) insiste en que este cambio metodológico en la docencia universitaria no es algo revolucionariamente nuevo. Las competencias siempre han formado parte de los currículos. Se trata más bien de una "nueva mirada", de una nueva manera de entender la educación, en la que se pasa de una fragmentación de conocimientos disciplinares a un modelo holístico donde todos los conocimientos convergen. Para ello será necesaria una excelente coordinación entre los docentes y aquellos que tomen el relevo (Marimón et al. 2009). Además, las competencias deberían ser objeto de revisión cada cierto número de años para asegurar un sistema académico más flexible y una mayor adaptabilidad a las nuevas realidades emergentes. Ahora bien, esta revisión siempre debe partir de la base de que el competente lingüístico por sí solo no es suficiente, por lo que resulta necesario integrarlo con el resto de habilidades y conocimientos que subyacen a la competencia traductora. Así pues, el conjunto de competencias que componen este modelo debe interpretarse desde un punto de vista global y no como segmentos separados.

López Ropero y Tabuenca (2009) afirman que uno de los mayores desafíos al que se enfrentan las titulaciones de Traducción e Interpretación es la baja competencia lingüística con la que ingresan los nuevos alumnos. De ahí la necesidad de investigación y establecimiento de un sistema de competencias sólido que ayude a dar respuestas metodológicas afines a esta enseñanza. Los traductores necesitan poseer una identidad propia que los distinga de otros usuarios de la lengua y la CT es la base de dicha identidad. Por esta razón, en el presente trabajo la sistematización de competencias se ha hecho a partir de un modelo de CT. Las asignaturas de lengua extranjera, al igual que el resto de asignaturas que compongan el plan de estudios de la titulación, deben contribuir al desarrollo de esta competencia madre, aunque, dependiendo de la asignatura, la incidencia en una subcompetencia u otra variará ligeramente.

Al hilo de la argumentación presentada al inicio del trabajo, hay que destacar que el modelo presentado se adapta fielmente a los principios competenciales que se establecen en el Marco Común Europeo de Referencia para 
las Lenguas (MCER). Además de las competencias clásicas de comprensión y expresión orales y escritas, por primera vez el MCER establece una competencia de mediación que permita a los usuarios de la lengua la comunicación entre hablantes de lenguas distintas que sean incapaces de comunicarse. Se trata de una habilidad de la que los primeros paradigmas lingüísticos se hacían eco en sus métodos, pero que, con la introducción de otros métodos de corte más comunicativo, quedó relegada a un segundo plano. Esta habilidad, no obstante, es la matriz sobre la que se basa el modelo presentado por estar dirigido a traductores e intérpretes en formación. Además, en la propuesta que aquí se presenta se aboga por una integración de las distintas competencias, lo cual también responde a los principios competenciales básicos recogidos en el MCER.

Por otra parte, la propuesta permite enmarcar esta subdisciplina dentro de la rama de lenguas con fines específicos. El objetivo es la preparación lingüística para la traducción. En la subcompetencia lingüística y comunicativa se propone el uso de textos tanto generales como especializados, así como el uso de textos paralelos. Además, se hace hincapié en el conocimiento de géneros textuales y de terminología especializada, así como en el uso de materiales de consulta de distinta índole. Incluye, además, la subcompetencia de transferencia como elemento metodológico clave que diferencia este tipo de docencia de otra con fines más generalistas.

Finalmente, cabe destacar que la limitación de este trabajo viene marcada mayoritariamente por la falta, hasta la fecha, de estudios empíricos específicos hasta la fecha que ayuden a contrastar el conjunto de competencias propuestas desde una perspectiva científica. La gran mayoría de los estudios existentes son de tipo descriptivo y se fundamentan en principios metodológicos generales derivados de la pedagogía de la traducción. De ellos no se desprenden datos cuantitativos y/o cualitativos que ayuden a cimentar los principios metodológicos que deben regir la enseñanza de lenguas extranjeras dentro de un título de Traducción e Interpretación. El presente estudio, no obstante, se fundamenta en un modelo de CT que sí ha sido sometido a un análisis empírico. Los resultados del análisis de PACTE ponen de manifiesto la pericia que caracteriza a un traductor e intérprete. El conjunto de competencias descritas en este trabajo se basa en este modelo de CT, por lo que parte de un modelo científicamente contrastado. No obstante, en nuestra propuesta, el modelo de CT ha sufrido ciertos cambios con el fin de adaptarlo al estadio de formación del alumnado. Así pues, en prospectiva, resultará necesario someter esta propuesta a un estudio de corte longitudinal que permita verificar las competencias 
dentro de la formación lingüística de futuros traductores e intérpretes y adaptar la propuesta en función de los resultados obtenidos.

\section{Referencias bibliográficas}

Almagro Esteban, Ana. (2001) "Rasgos que diferencian la enseñanza de ESP de la enseñanza de inglés general: ¿una metodología diferente?” En: Posteguillo, Santiago; Inmaculada Fontanet \& Juan Carlos Palmer (eds.) 2001. Methodology and New Technologies in Languages for Specific Purposes. Castellón de la Plana: Publicaciones de la Universitat Jaume I.

Andreu, Maribel; Laura Berenguer; Pilar Orero \& Odile Ripoll. (2002) "Competència traductora i ensenyament de llengües estrangeres." Quaderns: Revista de Traducció 7, pp. 155-165.

BEEBY, Allison. (1996) "Designing a foreign language course for trainee translators." Quaderns: Revista de Traducció 10, pp. 41-60.

BERENGUER ESTELLES, Laura. (1997) L'ensenyament de llengües estrangeres per a traductors. Didàctica de l'alemany. Barcelona: Universidad Autónoma de Barcelona. Tesis doctoral.

Bloomfield, Leonard. (1933) Language. New York: Henry Holt.

Bolívar, Antonio. (2008) "El discurso de las competencias en España: educación básica y educación superior." Red U. Revista de Docencia Universitaria 11. Número monográfico: Formación centrada en competencias (II).

Bolívar, Antonio. (2007) "La planificación por competencias en la reforma de Bolonia de la educación superior: un análisis crítico." Educaçao Telemática Digital 9, pp. 68-94.

Bowen, David \& Margareta Bowen. (1984) Steps to Consecutive Interpretation. Washington D.C.: Pen \& Booth.

BREHM CRIPPS, Justine. (2007) Targeting the Source Text: A Coursebook in English for Translator Trainees. Castellón de la Plana: Publicaciones de la Universidad Jaume I.

BReHM CRIPPS, Justine \& Amparo Hurtado Albir. (1999) "La primera lengua extranjera." En: Hurtado Albir, Amparo (eds.) 1999. Enseñar a traducir. Metodología en la formación de traductores e intérpretes. Madrid: Edelsa. Colección de investigación didáctica.

CANO GARCÍA, Ma Elena. (2008) "La evaluación por competencias en la educación superior." Profesorado. Revista de currículum y formación del profesorado 13:2, pp. 1-16.

Cerezo Herrero, Enrique. (2017) "A Critical Review of Listening Comprehension in Interpreter Training: The Case of Spanish Translation and Interpreting Degrees." Porta Linguarum 28, pp. 7-22. 
Cerezo Herrero, Enrique. (2015) "English Language Teaching for Translator and Interpreter Trainees: Syllabus Analysis and Design." Quaders: Revista de Traducció 22, pp. 289-306.

Cerezo Herrero, Enrique. (2013) La enseñanza del inglés como Lengua B en los estudios de Traducción e Interpretación en España: La comprensión oral para la interpretación. Valencia: Universitat de València. Tesis doctoral.

Clouet, Richard. (2010) Lengua inglesa aplicada a la traducción. Una propuesta curricular adaptada al Espacio Europeo de Educación Superior. Granada: Comares.

ColinA, Sonia. (2002) "Second Language Acquisition, Foreign Language Teaching and Translation Studies." The Translator 8:1, pp. 1-24.

CONSEJO DE EuRopa. (2001) Marco Común Europeo de Referencia para las Lenguas: Aprendizaje, Enseñanza, Evaluación. Instituto Cervantes.

CRuz García, Laura \& Maureen Mulligan. (2004) "The Teaching of English as a Foreign Language for Translators: A Theoretical Approach." Third International Conference. Language as a Means for Education, Research and Professional Development. Varna University, Bulgaria.

De Miguel DíAZ, Mario. (coord.) (2006) Metodologías de enseñanzas y aprendizaje para el desarrollo de competencias: orientaciones para el profesorado universitario ante el Espacio Europeo de Educación Superior. Madrid: Alianza Editorial.

DelisLe, Jean. (1993) La traduction raisonnée. Ottawa: Les Presses de l'Université d'Ottawa.

DeLISLE, Jean. (1988) Translation: An Interpretative Approach. Ottawa: University of Ottawa Press.

DelisLe, Jean. (1980) Lanalyse du discours comme méthode de traduction. Ottawa, University of Ottawa Press.

Duff, Alan. (1981) The Third Language. Oxford: The Pergamon Press.

FueNTES LuQUE, Adrián. (2001) "Traducción diplomática, que no diplomacia en la traducción." En: Kelly, Dorothy (eds.) La traducción y la interpretación en España hoy: perspectivas profesionales. Granada: Comares.

GONZÁlEZ, Julia \& Robert WAGENAAR. (2003) Tuning Educational Structures in Europe. Bilbao: University of Deusto. Final Report.

Grosjean, François. (2010) Bilingual: Life and Reality. Cambridge: Harvard University Press.

HANSEN, Gyde. (1997) "Success in translation." Perspectives: Studies in Translatology 5: 2, pp. 201-210.

HARRIS, Brian \& Bianca SHERWOOD. (1978) “Translating as an Innate Skill.” En: Gerver, David \& Wallace Sinaiko (eds.) 1978. Language, Interpretation and Communication. Oxford: Plenum Press, pp. 155-170.

HATIM, Basil \& Iam MASON. (1990) Discourse and The Translator. London: Longman. 
HeWSOn, Lance \& Jacky MarTin. (1991) Redefining Translation. The Variational Approach. London \& New York: Routledge.

Hurtado Albir, Amparo. (2017) "Translation and Translation Competence." En: Hurtado Albir, Amparo (eds.) Researching Translation Competence by PACTE Group. Amsterdam: John Benjamins.

HurTado Albir, Amparo. (2011) Traducción y Traductología. Introducción a la Traductología. Madrid: Cátedra.

Hurtado Albir, Amparo. (1999a) "Objetivos de aprendizaje y metodología en la formación de traductores e intérpretes." En: Hurtado Albir, Amparo (eds.) La enseñanza de la traducción. Castellón de la Plana: Publicaciones de la Universidad Jaume I.

HurTAdo Albir, Amparo. (1999b) "La competencia traductora y su adquisición. Un modelo holístico y dinámico." Perspectives: Studies in Translatology 7: 2, pp. 177-188.

IMBERnON MuÑoz, Fransesc \& José Luis Medina MoYA. (2005) Metodologia participativa a l'aula universitària. La participación de l'alumnat. Barcelona: ICE.

KELLY, Dorothy. (2002) "Un modelo de competencia traductora: bases para el diseño curricular." Puentes 1, pp. 9-20.

Kiraly, Donald. (2000) A Social Constructivist Approach to Translator Education. Empowerment from Theory to Practice. Manchester: St. Jerome.

Kiraly, Donald. (1995) Pathways to Translation. Pedagogy and Process. Kent, OH: The Kent State University Press.

Krashen, Stephen. (1982) Principals and Practice in Second Language Acquisition. New York: Pergamon Press.

LAM, Agnes. (2001) "Bilingualism." En: Carter, Ronald \& David Nunan (eds.) 2001. The Cambridge Guide to Teaching English to Speakers of Other Languages. Cambridge: Cambridge University Press, pp. 93-99.

Le BOterf, Guy. (2000) Ingeniería de las competencias. Barcelona: Gestión 2000.

López Ropero, Lourdes \& M. Felicidad Tabuenca Cuevas. (2009) "Lengua B1 (Traducción e Interpretación) y competencias.” En: Gómez Lucas, Cecilia \& Salvador Grau Company (eds.) 2009. Propuestas de diseño, desarrollo e innovaciones curriculares y metodología en el EEES. Alcoy: Marfil, pp. 121-134.

LÓPEZ RUIZ, Juan Ignacio. (2011) "Un giro copernicano en la enseñanza universitaria: formación por competencias." Revista de Educación 356, pp. 279-301.

MACKEnZIE, Rosemary. (1998) "The Place of Language Teaching in Quality Oriented Translators' Training Programme." En: Malmkjaer, Kristen (eds.) 1998. Translation and language teaching. Manchester: St. Jerome.

MACKEY, William F. (1970) A typology of bilingual education. Foreign Language Annals.

MARIMÓN LlORCA, Carmen et al. (2009) "La elaboración de guías docentes para el primer curso de las filologías: de las competencias al diseño de objetivos." 
En: Tortosa Ybáñez, María Teresa \& José Daniel Álvarez Teruel (coord.) Investigaciones colaborativas en el ámbito universitario: propuestas para el cambio. Alicante: Universidad de Alicante.

Mayoral ASEnSIO, Roberto. (2001) "Por una renovación en la formación de traductores e intérpretes: revisión de algunos de los conceptos sobre los que basa el actual sistema su estructura y contenidos." Sendebar 10/11, pp. 67-88.

MÖLler Runge, Julia. (2001) Siglo XXI. ¿Innovamos? La enseñanza de una segunda lengua extranjera. Salobreña: Alhulia.

MORIN, Edgar. (2001) Los siete saberes necesarios para la educación del futuro. Barcelona: Seix Barral.

NeUbERT, Albrecht. (2000) "Competence in Translation, in Languages, and in Translation.” En: Schäffner, Christina \& Beverly Abad (eds.) Developing Translation Competence. Amsterdam: John Benjamins.

NeUBERT, Albrecht. (1994) "Competence in Translation: a complex skill, how to study and how to teach it." En: Snell-Hornby, Mary; Franz Pöchhacker \& Klaus Kaindl (eds.) 1994. Translation Studies: An Interdiscipline. Amsterdam: John Benjamins.

OSTER, Ulrike. (2008) "El reto de enseñar la Lengua C en la titulación de Traducción e Interpretación (antes y después de Bolonia)." Jornada nacional sobre estudios universitarios, Universidad Jaime I.

PACTE. (2017) Researching Translation Competence by PACTE Group. Amsterdam: John Benjamins.

PACTE. (2011) "Results of the Validation of the PACTE Translation Competence Model: Translation Project and Dynamic Translation Index." En: O'Brien, Sharon (eds.) 2011. IATIS Yearbook 2010. London: Continuum.

PÉREZ Gómez, Ángel I. (2007) La naturaleza de las competencias básicas y sus aplicaciones pedagógicas. Santander: Gobierno de Cantabria, Conserjería de Educación.

Peterson, Elizabeth \& Brownen Coltrane. (2003) "Culture in Second Language Acquisition." CAL Digest (EDO-FL-03-09). Centre for Applied Linguistics.

PRESAS, Marisa. (2003) "Competència bilingüe vs. Competència traductora." VII Jornadas de Traducción en Vic.

PRIETO NAVARRO, Leonor (coord.) (2008) La enseñanza universitaria centrada en el aprendizaje: Estrategias útiles para el profesorado. Barcelona: Octaedro/ICE UB.

PyM, Anthony. (1992) "Translation error analysis and the interface with language teaching." En: Dollerup, Cay \& Anne Loddegaard (eds.) 1992. Teaching Translation and Interpreting: Training, Talent and Experience. Amsterdam: John Benjamins, pp. 279-288.

REAL DECRETO 1393/2007, de 29 de octubre, por el que se establece la ordenación de las enseñanzas universitarias oficiales. 
REISS, Katharina \& Hans J. VermeER. (1991) Grundlegung einer allgemeine Translationstheorie. Tübingen: Niemeyer.

RISKU, Hanna. (1998) Translatorische Kompetenz. Kognitive Grundlagen des Übersetzens als Expertentätigkeit. Tübingen: Stauffenburg.

ROBERTS, Roda P. (1984) "Compétence du nouveau diplômé en traduction." En: Traduction et Qualité de Langue, Actes du Colloque, Société des traducteurs du Québec/Conseil de la langue française. Québec: Éditeur officiel du Québec, pp. 172-184.

SELESKOVITCH, Danica \& Marianne LEDERER. (1984) Interpréter pour traduire. Paris: Didier Érudition. Colección Traductologie I.

Snell-Hornby, Mary. (1988) Translation Studies. An Integrated Approach. Amsterdam \& Philadelphia: John Benjamins.

SORIANO GARCÍA, Inmaculada. (2004) "La enseñanza de lenguas en la formación de traductores: modelo de lecturas en casa." Actas del IX Seminario Hispano-Ruso de Traducción e Interpretación. Moscú: MGLU, pp. 151-159.

StALMACH PAJESTKA, Jadwiga. (2008) "La enseñanza de la lengua extranjera orientada a la traducción: el caso de la lengua C (Ruso)." En: Pascual Febles, Isabel (eds.) 2008. La formación de traductores e intérpretes. Aproximación interdisciplinar. Gran Canaria: Servicio de publicaciones de la Universidad de las Palmas de Gran Canaria.

Titone, Renzo. (1972) Bilinguismo prococe ed educazione bilingüe. Roma: Armando Ed.

VARIOS Autores. (Agencia Nacional de Evaluación de la Calidad y Acreditación). (2004) Libro blanco. Título de grado en Traducción e Interpretación.

VINAY, Jean Paul \& Jean Darbelnet. (1970) Stylistique comparée du français et de l'anglais. Méthode de traduction. Paris: Didier.

WILSS, Wolfram. (1976) "Perspectives and limitations of a didactic framework for the teaching of translation." En: Brislin, Richard W. (eds.) Translation Applications and Research. New York: Gardner, pp. 117-137.

WiLSS, Wolfram. (1982) The Science of Translation: Problems and Methods. Tübingen: Gunter Narr.

YÁNIZ, Concepción (2006) "Planificar la enseñanza universitaria para el desarrollo de las competencias." Educatio XXI 24, pp. 17-34. 


\section{BIONOTA / BIONOTE}

ENRIQUe CEREZo Herero es Doctor en Traducción e Interpretación por la Universitat de València. Su tesis versó sobre la enseñanza de inglés en la formación de traductores e intérpretes. Sus principales líneas de investigación son la enseñanza y adquisición de lenguas extranjeras, la pedagogía de la traducción e interpretación y la educación bilingüe. Actualmente es profesor de lengua, lingüística y didáctica inglesas en la Universidad CEU Cardenal Herrera. Es además el director del Máster Universitario en Educación Bilingüe en esta misma universidad, impartiendo asignaturas de metodología y fundamentos sobre bilingüismo.

ENRIQUe CEREZO HERRERO holds a PhD in Translation and Interpreting from the University of València. His dissertation dealt with the teaching of English within Translation and Interpreting studies. His main research areas are: the teaching and acquisition of foreign languages, translation pedagogy and bilingual education. Currently, he is a lecturer of English language, linguistics and didactics at the University CEU Cardenal Herrera. Additionally, he is the director of the Master's Degree in Bilingual Education at the same university, teaching subjects related to methodology and foundations of bilingual education. 
